\title{
Assessment of myocardial viability: an effective gatekeeper for coronary revascularization?
}
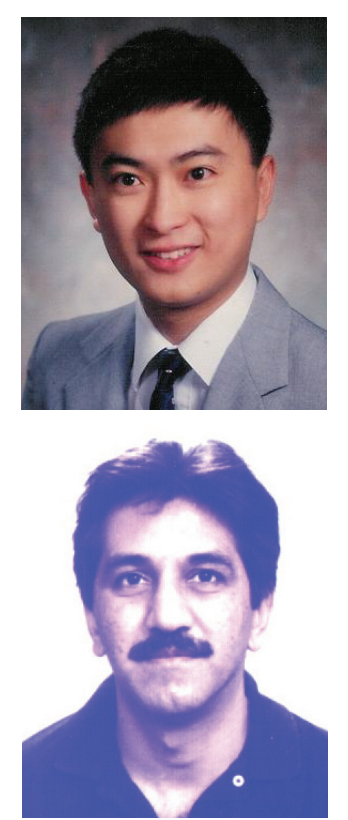

Siu-Sun Yao \& Farooq A Chaudhry ${ }^{\dagger}$

${ }^{\dagger}$ Author for correspondence St Luke's-Roosevelt Hospital Center, Columbia University College of Physicians \& Surgeons, New York, USA Tel.: + 1212523 4296; Fax: +1 212523 5989; fchaudhr@chpnet.org

\section{'... approximately $50 \%$ of patients diagnosed with heart failure will be dead within 5 years.'}

Heart failure is an emerging worldwide epidemic. In the USA alone, there are an estimated 4.8 million Americans with heart failure, $10 \%$ of persons aged 70 years or greater and 400,000 new cases annually [1]. Heart failure is the most common diagnosis in hospitalized patients aged 65 years and older. There is an exceedingly high mortality rate associated with heart failure; approximately $50 \%$ of patients diagnosed with heart failure will be dead within 5 years. As the population ages, with more cardiac patients living longer with their disease, the opportunity for developing heart failure increases. The economic costs of heart failure are substantial with an estimated US $\$ 23.2$ billion society costs per year in the USA.

Coronary artery disease (CAD) is the most common cause of left ventricular (LV) systolic dysfunction leading to heart failure [2]. It was previously thought that loss of myocardial contractility after myocardial infarction was caused by the destruction of myocardium and the development of irreversible scarring. With the subsequent development of coronary artery bypass grafting (CABG), clinical observations showed that in some individuals, the function of hypo or akinetic segments could improve following revascularization [3]. The contractile regional dysfunction seen in patients may be either transient (stunning) or prolonged (hibernation). In reality, the spectrum of myocardial dysfunction is probably a continuum extending from myocardial ischemia to programmed cell death and apoptosis. A proportion of patients with heart failure will confer viability by symptomatic angina or ischemia demonstrated by myocardial perfusion imaging [4]. However, most patients with LV systolic dysfunction due to CAD have a heterogeneous mixture of stunned, hibernating and scarred myocardium in various proportions.

Given many considerations, it is clear that patients with heart failure due to CAD should pursue treatment options to prevent future coronary events. These therapies include treatment of heart failure, myocardial ischemia, and myocardial stunning and hibernation. Standard treatment of patients with heart failure due to LV systolic dysfunction include angiotensin-converting enzyme (ACE) inhibitors, angiotensinreceptor blockers, $\beta$-blockers and aldosterone antagonists [5-8]. Many cardiologists, and some guidelines [9], recommend evaluating patients with CAD and heart failure to identify patients suitable for coronary revascularization, although this strategy is not evidence based. It is often believed that the presence of viable but dysfunctional myocardium is an indication for revascularization, although very few patients with heart failure undergo viability testing.

\section{Evaluation of hibernating myocardium}

Transient postischemic dysfunction is termed 'stunned' myocardium. Hibernating myocardium is defined as a region of depressed contractility at rest, but which is potentially reversible owing to chronically impaired coronary blood flow and a stenosed coronary artery. However, several observations suggest that the difference between stunning and hibernation may be one of degree: in stunning, resting blood flow is normal but maximal blood flow is reduced, whereas in hibernation, resting blood flow is low, leading to the hypothesis that hibernation may result from repetitive or chronic stunning.

'The principal goal of myocardial viability assessment is to identify patients whose natural history of ischemic heart failure may improve after revascularization.

It has been estimated that $20-50 \%$ of patients with chronic ischemic LV dysfunction have a significant amount of viable hibernating myocardium and, therefore, the potential for meaningful clinical improvement in LV function following successful revascularization [10].

The principal goal of myocardial viability assessment is to identify patients whose natural history of ischemic heart failure may improve after revascularization. Noninvasive imaging techniques [11] can be used to detect myocardial viability by 
demonstrating cell membrane integrity (thallium201 imaging), inotropic contractile reserve (dobutamine stress echocardiography and MRI), intact mitochondrial function (technetium-99m sestamibi imaging), preserved myocardial metabolism (PET with F-18 deoxyglucose), absence of scar tissue in areas of dysfunctional myocardium (gadolinium-enhanced MRI) or microvascular integrity (myocardial contrast echocardiography).

'The strongest predictor of

outcome is revascularization of ischemic LV segments...'

Assessment of viability is most relevant in patients with dyspnea rather than angina. Noninvasive imaging may be considered to define the likelihood of CAD (myocardial ischemia and viability) in patients with heart failure and LV dysfunction (myocardial perfusion defects, deterioration of wall motion abnormalities or biphasic response). Thallium-201 imaging and dobutamine echocardiography are similar in accuracy for the detection of viable myocardium. The former may have increased sensitivity; the latter higher specificity. Differences in viability assessment between dobutamine echocardiography and Thallium-201 or PET imaging may reflect underlying alternations in cellular metabolism and function. The 'overestimation' of viability with nuclear imaging techniques may result from the detection of small regions of viability that are of inadequate size to permit improvement in regional or global systolic function. A heterogeneous admixture of necrotic and viable cells may not demonstrate improved LV contraction, in spite of the presence of adequate metabolic function in at least some of the myocytes. The ultimate choice in imaging technique may depend upon local availability and laboratory expertise. PET with F-18 deoxyglucose or gadolinium-enhanced MRI may be performed for clarification of nondiagnostic or equivocal prior testing.

\section{Risk stratification \& prognosis Medical therapy for heart failure}

The improvement in LV ejection fraction (LVEF) in response to $\beta$-blockers is heterogeneous in patients with ischemic heart failure, possibly indicating variations in the myocardial substrate underlying LV dysfunction. The Carvedilol Hibernation Reversible Ischemia Trial (CHRISTMAS) investigated whether the improvement in LVEF was associated with the volume of hibernating viable myocardium with contractile reserve [12]. Hibernation was identified by a mismatch between echocardiographic regional wall motion and nitrate-enhanced technetium-99m sestamibi perfusion. The study was a multicenter, double-blind, randomized trial to compare placebo and carvedilol for 6 months in 489 patients with chronic stable heart failure due to ischemic LV systolic dysfunction (mean LVEF $=30 \pm 11$ ). The primary end point was change in LVEF (measured by radionuclide ventriculography), in nonhibernators and hibernators and in those taking carvedilol compared with placebo. There was a high prevalence $(59 \%)$ of patients classified as hibernators. LVEF was unchanged with placebo, but increased with carvedilol in both patients without (2.5\%) and with (3.2\%) hibernating myocardium. Patients with more hibernating myocardium or ischemia had a greater increase in LVEF on carvedilol. The study concluded that medical treatment with carvedilol may be an important adjunct or alternative to revascularization for patients with hibernating myocardium.

\section{'...the number of dysfunctional segments demonstrating contractile reserve was the strongest predictor of survival ... but this survival advantage was not maintained beyond 3 years.'}

The CHRISTMAS trial was not powered to demonstrate a difference in mortality rate. The implication from the CHRISTMAS trial is that the quantity of hibernating myocardium is important in determining the improvement in LVEF with $\beta$-blocker treatment. However, it is important to note that even patients with no hibernating myocardium benefited from $\beta$-blockers.

\section{Revascularization for heart failure}

The identification of viable myocardium with dobutamine echocardiography provides important prognostic information in patients with LV dysfunction. The presence of inotropic contractile reserve with low-dose dobutamine echocardiography predicts a better initial survival compared with the absence of inotropic contractile reserve, independent of symptoms, baseline LV function or coronary anatomy. The strongest predictor of outcome is revascularization of ischemic LV segments, followed by that of viable LV segments. 
In a study by Chaudhry et al. the prognostic implications of myocardial contractile reserve were evaluated in patients with CAD and LV dysfunction [13]. Dobutamine echocardiography was performed in 80 patients with CAD and LV dysfunction (ejection fraction: 40\%) and serial prospective follow-up was obtained in all patients (mean follow-up: $2.2 \pm 1.1$ years). By multivariate analysis, the number of dysfunctional segments demonstrating contractile reserve was the strongest predictor of survival $(\mathrm{p}<0.03)$.

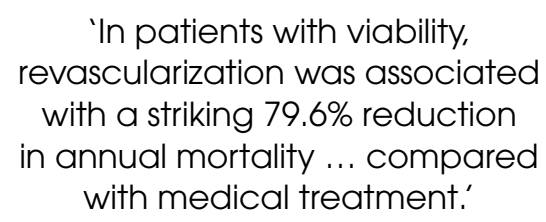

Patients with contractile reserve had better initial survival during medical therapy than did those without contractile reserve, but this survival advantage was not maintained beyond 3 years. By contrast, survival was excellent in patients with contractile reserve who underwent myocardial revascularization. Among 58 patients with contractile reserve in five myocardial segments, survival at 3 years was $93 \pm 6 \%$ in the 24 patients who were revascularized, but only $49 \pm 15 \%$ in the 34 treated medically $(\mathrm{p}<0.02)$. Thus, myocardial contractile reserve is a significant predictor of survival in patients with CAD and LV dysfunction undergoing medical therapy, independent of symptoms, baseline LV function or coronary anatomy. Survival in patients with significant contractile reserve is further enhanced by surgical revascularization.

Allman et al. later summarized 24 trials in a meta-analysis of 3088 patients with chronic CAD and LV dysfunction (mean LVEF: $=32 \pm 8$ ) undergoing different techniques for identifying hibernating myocardium between 1966 and August 1999 [10]. In patients with viability, revascularization was associated with a striking $79.6 \%$ reduction in annual mortality (16 vs $3.2 \%$; $\mathrm{p}<0.0001)$ compared with medical treatment. As the severity of LV dysfunction increased, the potential survival benefit associated with revascularization of patients with viability also increased. Evidence of viability may provide information on potential clinical benefit of revascularization with worsening $\mathrm{LV}$ dysfunction to balance against an increasing procedural risk. There is no apparent outcome benefit of revascularization in the absence of demonstrated viability, and there is a trend toward higher mortality with revascularization. Most studies suggest that between a third to a half of total myocardial mass needs to be affected before a survival benefit from revascularization is seen [14].

The current level of evidence regarding the use of viability imaging to guide decision-making has limitations. For example, although viability imaging is predicated on the ability to predict functional recovery of myocardium, the failure to improve LV function after revascularization is not necessarily associated with a worse clinical outcome, presumably because revascularization of ischemic myocardium may be crucial for longterm prognosis. Effective revascularization may promote resolution of ongoing inflammation, prevent tissue injury and fibrosis, infarct expansion, ventricular remodeling, ventricular arrhythmias and the development of heart failure $[15,16]$. In fact, there also appears to be a 'critical window' of time for revascularization since there is a progressive diminution of chance for complete structural and functional recovery after restoration of blood flow and subsequent nonviability [17].

Medical therapy has also advanced since the meta-analysis was conducted. In addition to angiotensin-converting enzyme inhibitors, spironolactone, $\beta$-blockers and aldosterone antagonists have been shown to be effective for heart failure [5-8]. This leaves open the question of whether revascularization is superior to current best medical therapy.

\section{'Most studies suggest that between a third to a half of total myocardial mass needs to be affected before a survival benefit from revascularization is seen.'}

Meta-analyses may also greatly oversimplify an extremely complex issue (myocardial viability). Meta-analyses are subject to publication bias, which may exclude unpublished or unlocated studies and systematically exclude papers written in languages other than English. LeLorier et al. previously reported that the outcomes of 12 large, randomized, controlled trials were not predicted accurately $35 \%$ of the time by the meta-analyses published previously on the same topics [18].

\section{Outcome studies: HEART \& STICH}

The Heart Failure Revascularization Trial (HEART) is a multicenter study of 800 patients with heart failure, a LVEF of less than $35 \%$ and evidence of CAD who are receiving optimal medical treatment followed for 5 years. The main aim of the study is to determine whether coronary 
revascularization improves the survival of patients with heart failure due to CAD who have evidence of dysfunctional but viable myocardium or stressinduced ischemia [19]. Secondary end points include the effects of revascularization on nonfatal vascular events, hospitalization, symptoms of heart failure and angina, quality of life and health economic outcomes. In 2002, the National Heart, Lung, and Blood Institute (NHLBI) funded a similar multicenter international trial of 2000 patients, the Surgical Treatment for Ischemic Heart Failure (STICH) trial [20], to address the optimal management of patients with heart failure and decreased LV function with CAD suitable for revascularization:

- Is CABG surgery superior to contemporary medical/secondary prevention therapy in prolonging survival in these patients?

- Does the addition of surgical ventricular reconstruction to CABG among patients with significant anterior wall dysfunction improve hospitalization-free survival?

Secondary end points will examine quality of life, cost-effectiveness and changes in LV volumes, effect of myocardial viability, selected biomarkers, and selected polymorphisms on treatment differences. Only revascularization by CABG is permitted in the STICH protocol. This may lead to higher procedural risk than in the HEART trial, but could also result in longer sustained gains. The primary outcomes of the STICH trial are anticipated by 2009 (hypothesis 2) and 2011 (hypothesis 1). Since revascularization in patients with LV systolic dysfunction is associated with substantial risk, the HEART and STICH trials will help determine when and how viability imaging is used to predict long-term outcomes.

\section{Conclusion}

Hibernating myocardium is functionally dormant but still viable. The CHRISTMAS trial showed that the quantity of viable myocardium was likely to govern the response to medical therapy with $\beta$-blocker medications. The exact role of identifying hibernating myocardium by viability assessment techniques in order to recommend revascularization in those most likely to benefit remains unclear. Many trials have suggested that revascularization is preferable to medical therapy when there is significant myocardial viability.

It is disputable whether the recovery of functional myocardium following revascularization is the best gold standard predictive of long-term prognosis. Since coronary bypass is beneficial in patients with multivessel CAD and LV dysfunction, it could be argued that coronary revascularization should be considered in all patients with ischemic cardiomyopathy, whether or not there is evidence of viable myocardium. This is substantiated by the fact that $10-15 \%$ of patients with ischemic cardiomyopathy will improve LV function, despite a negative noninvasive study (80-90\% sensitivity) demonstrating no viability.

Given limited healthcare resources and significant risks of revascularization, the prospective HEART and STICH trials will help determine whether revascularization of patients with heart failure and LV systolic dysfunction secondary to $\mathrm{CAD}$ is safe or beneficial in the presence of myocardial viability.

'... current literature would support ... the consideration of revascularization before LV dysfunction may become irreversible.'

The studies will also address how valuable are current imaging modalities for predicting candidates for medical or surgical revascularization. Is revascularization effective in prolonging survival in patients with heart failure who have no symptoms of angina? What is the role of current imaging modalities for viability assessment in predicting candidates for medical or surgical management? Is there a clinical benefit of surgical revascularization? We eagerly await the answers to these and many other related questions.

In the interim, current literature would support the importance of identifying viable myocardium and LV dysfunction, and the consideration of revascularization before LV dysfunction may become irreversible [17]:

- LV dysfunction with ischemia: revascularization is beneficial;

- LV dysfunction with ischemia and significant (>60\%) viable myocardium: revascularization is beneficial;

- LV dysfunction with moderate (30-50\%) viable myocardium: revascularization is probably beneficial;

- LV dysfunction with minimal (<30\%) viable myocardium: revascularization is probably not beneficial;

- LV dysfunction with inotropic contractile reserve: optimal medical treatment with $\beta$-blockers, ACE inhibitors and angiotensinreceptor blockers. 
Financial \& competing interests disclosure Farooq A Chaudhry received a grant from POINT Biomedical and Lantheus, and is also on the Speakers' Bureau for Lantheus. The authors have no other relevant affiliations or financial involvement with any organization or entity with a financial interest in or financial conflict with the subject matter or materials discussed in the manuscript. This includes employment, consultancies, honoraria, stock ownership or options, expert testimony, grants or patents received or pending, or royalties.

No writing assistance was utilized in the production of this manuscript.

\section{Bibliography}

1. Ho KK, Pinsky JL, Kannel WB, Levy D: The epidemiology of heart failure: the Framingham Study. J. Am. Coll. Cardiol. 22(4), 6A-13A (1993).

2. Gheorgiade $\mathrm{M}$, Bonow RO: Chronic heart failure in the United States. A manifestation of coronary artery disease. Circulation 97 , 282-289 (1998).

3. Kloner RA, Bolli R, Marban E, Reinlib L, Braunwald E: Medical and cellular implications of stunning, hibernation, and preconditioning: an NHLBI workshop. Circulation 97, 1848-1867 (1998).

4. Pasquet A, Robert A, D'Hondt AM et al: Prognostic value of myocardial ischemia and viability in patients with chronic left ventricular ischemic dysfunction. Circulation 100(2), 141-148 (1999).

5. Pitt B, Zannad F, Remme WJ et al: The effect of spironolactone on morbidity and mortality in patients with severe heart failure. Randomized Aldactone Evaluation Study Investigators. N. Engl. J. Med. 341, 709-717 (1999).

6. The Cardiac Insufficiency Bisoprolol Study II (CIBIS-II): a randomized trial. Lancet 353, 9-13 (1999).

7. Hjalmarson A, Goldstein S, Fagerberg B et al.: Effects of controlled-release metoprolol on total mortality, hospitalizations, and well-being in patients with heart failure: the Metoprolol CR/XL Randomized Intervention Trial in congestive heart failure (MERIT-HF). MERIT-HF Study Group. JAMA 283, 1295-1302 (2000).

8. Pfeffer MA, McMurray JJV, Velazquez EJ et al:: Valsartan, captopril, or both in myocardial infarction complicated by heart failure, LV dysfunction, or both. N. Engl. J. Med. 349, 1893-1906 (2003).
9. Hunt SA, Baker DW, Chin MH et al: ACC/AHA Guidelines for the evaluation and management of chronic heart failure in the adult: executive summary. A report of the American College of Cardiology/American Heart Association Task Force on Practice Guidelines. Circulation 104(24), 2996 (2001).

10. Allman KC, Shaw LJ, Hachamovitch R et al.: Myocardial viability testing and impact of revascularization on prognosis in patients with coronary artery disease and LV dysfunction: a meta-analysis. J. Am. Coll. Cardiol. 39, 1151-1158 (2002).

11. Bax JJ, Wijns W, Cornel JH et al:: Accuracy of currently available techniques for prediction of functional recovery after revascularization in patients with left ventricular dysfunction due to coronary artery disease: comparison of pooled data. J. Am. Coll. Cardiol. 30, 1451-1460 (1997).

12. Cleland JG, Pennell DJ, Ray SG et al: Myocardial viability as a determinant of the ejection fraction response to carvedilol in patients with heart failure (CHRISTMAS trial): randomized controlled trial. Lancet 362, 14-21 (2003).

13. Chaudhry FA, Tauke JT, Alessandrini RS et al:: Prognostic implications of myocardial contractile reserve in patients with coronary artery disease and LV dysfunction. J. Am. Coll. Cardiol. 34, 730-738 (1999).

14. Senior R, Kaul S, Lahiri A: Myocardial viability on echocardiography predicts long-term survival after revascularization in patients with ischemic congestive heart failure. J. Am. Coll. Cardiol. 33, 1848-1854 (1999).

15. Frangogiannis NG, Shimoni S, Chang SM et al:: Evidence for an active inflammatory process in the hibernating human myocardium. Am. J. Pathol. 160, 1425-1433 (2002).
16. Samady H, Elefteriades JA, Abbott BG et al: Failure to improve LV function after coronary revascularization for ischemic cardiomyopathy is not associated with worse outcome. Circulation 100, 1298-1304 (1999).

17. Elsasser A, Schlepper M, Klovekorn WP: Hibernating myocardium: an incomplete adaptation to ischemia. Circulation 96, 2920-2931 (1997).

18. LeLorier J, Gregoire G, Benhaddad A et al.: Discrepancies between meta-analyses and subsequent large randomized, controlled trials. N. Engl. J. Med. 337, 536-542 (1999).

19. Cleland JGF, Freemantle SG, Ball RS et al:: The heart failure revascularization trial (HEART): rationale, design and methodology. Eur. J. Heart Fail. 5, 295-303 (2003).

20. Velazquez EJ, Lee L, O’Connor CM et al: The rationale and design of the surgical treatment for ischemic heart failure (STICH) trial. J. Thorac. Cardiovasc. Surg. 134(6), 1540-1547 (2007).

\section{Affiliations}

- Siu-Sun Yao, MD St Luke's-Roosevelt Hospital Center, Department of Medicine, Division of Cardiology, Columbia University College of Physicians o Surgeons, NY, USA Tel.: + 12124925590 ;

Fax: +12124925555 ; syao@chpnet.org

- Farooq A Chaudhry, MD, FACC, FACP, FAHA, FASE Associate Chief of Cardiology, Director of Echocardiography, Associate Professor of Medicine, St Luke's-Roosevelt Hospital Center, Columbia University College of Physicians \& Surgeons, NY, USA Tel.: +1 2125234298 ; Fax: +1 2125235989 ; fchaudhr@chpnet.org 